\title{
Use of histamine-2 receptor antagonists is associated with infection in newborns hospitalized, but not necrotizing enterocolitis and mortality: A systematic review and meta-analysis
}

Ricardo Queiroz Gurgel, Ruth Nogueira da Silva Santana, Victor Santana Santos, Marina Santana Freire, Paulo Ricardo Saquete Martins Filho.

Graduate Program of Health Sciences and Department of Medicine. Federal University of Sergipe,

Aracaju, SE, Brazi

\section{Background}

Infections are the major cause of mortality in preterm newborns worldwide. It is well established that the gastric acid secretion is an important non immune defense barrier for infants against invading pathogens. The use of histamine-2 receptor inhibitors $\left(H_{2} R A\right)$ may predispose to infections and/or necrotizing enterocolitis (NEC) in neonates. We have conducted a systematic review and meta-analysis to investigate if the use of $\mathrm{H}_{2} R A$ by preterm newborns is associated or not with infection, NEC and mortality.

\section{Methods}

We performed a systematic review using PubMed, Web of Science and SCOPUS databases to identify studies published in Portuguese, Spanish or English up to April-2017. Publications were identified using the terms "histamine-2 recepto antagonists", "infection", "necrotizing enterocolitis", "mortality" and related terms. We calculated the pooled odds ratio (OR) for outcomes and a forest plot to present the effect sizes with 95\% confidence intervals $(95 \% \mathrm{Cl})$.

\section{Results}

The literature search identified 1,144 records. After screening titles and abstracts, 35 full-text articles were assessed for eligibility and nine were included. Five studies used a case-control design, four were cohorts and none was a clinical trial. Six studies involving 2,143 subjects were included neonates not exposed to $\mathrm{H}_{2} \mathrm{RA}$ resulting in a pooled OR of $3.44(95 \% \mathrm{Cl}$ : $1.49-7.92)$ (Figure 1)

\section{Figure 1. Results for infection outcome}

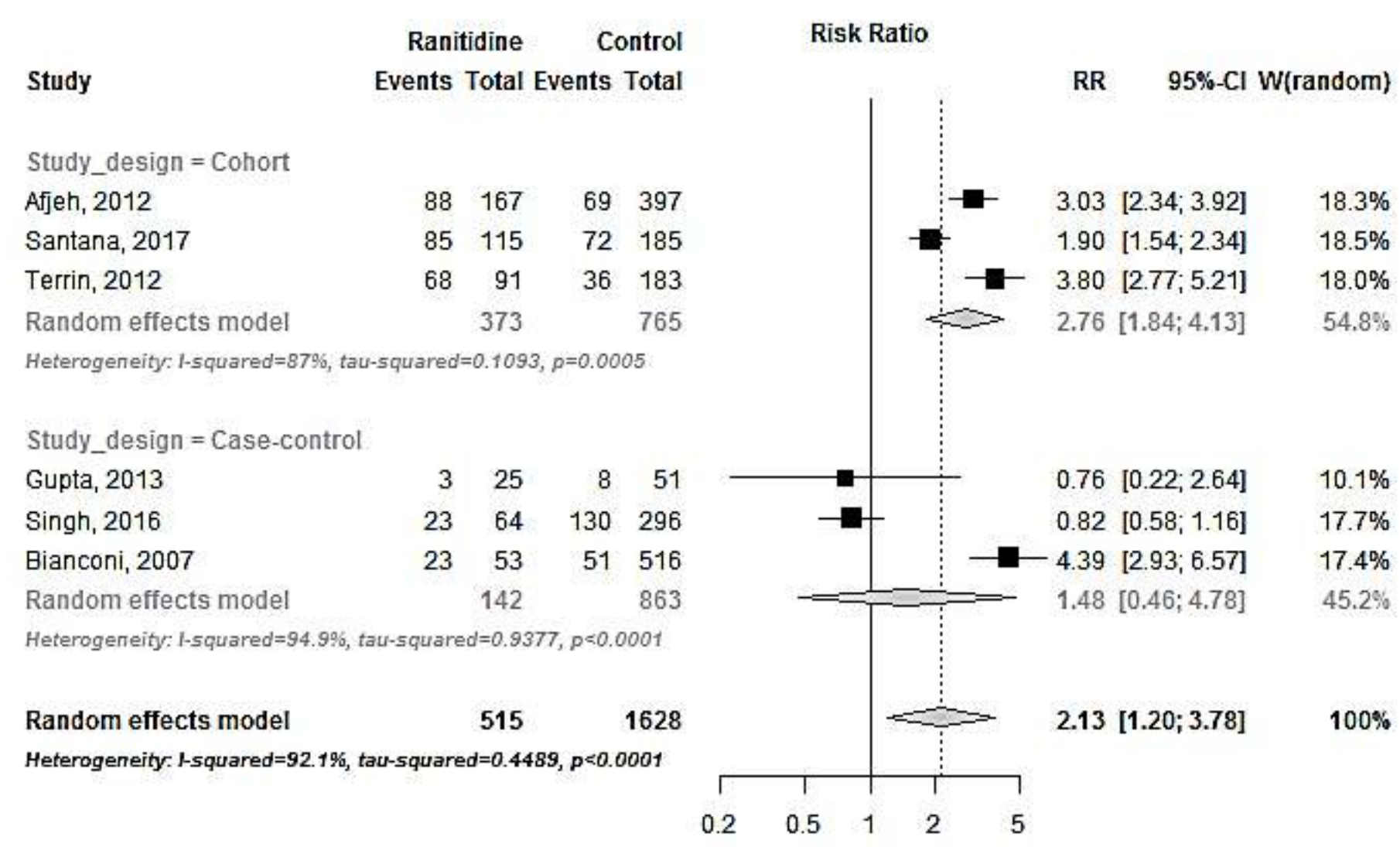

There was association between the use of $\mathrm{H} 2$ blockers and increased risk of infection. Unadjusted analyses from the three studies evaluating NEC showed that there was no association between the use of $\mathrm{H} 2$ blockers and this clinical condition (Figure 2).
Figure 2. Results for necrotizing enterocolitis outcome

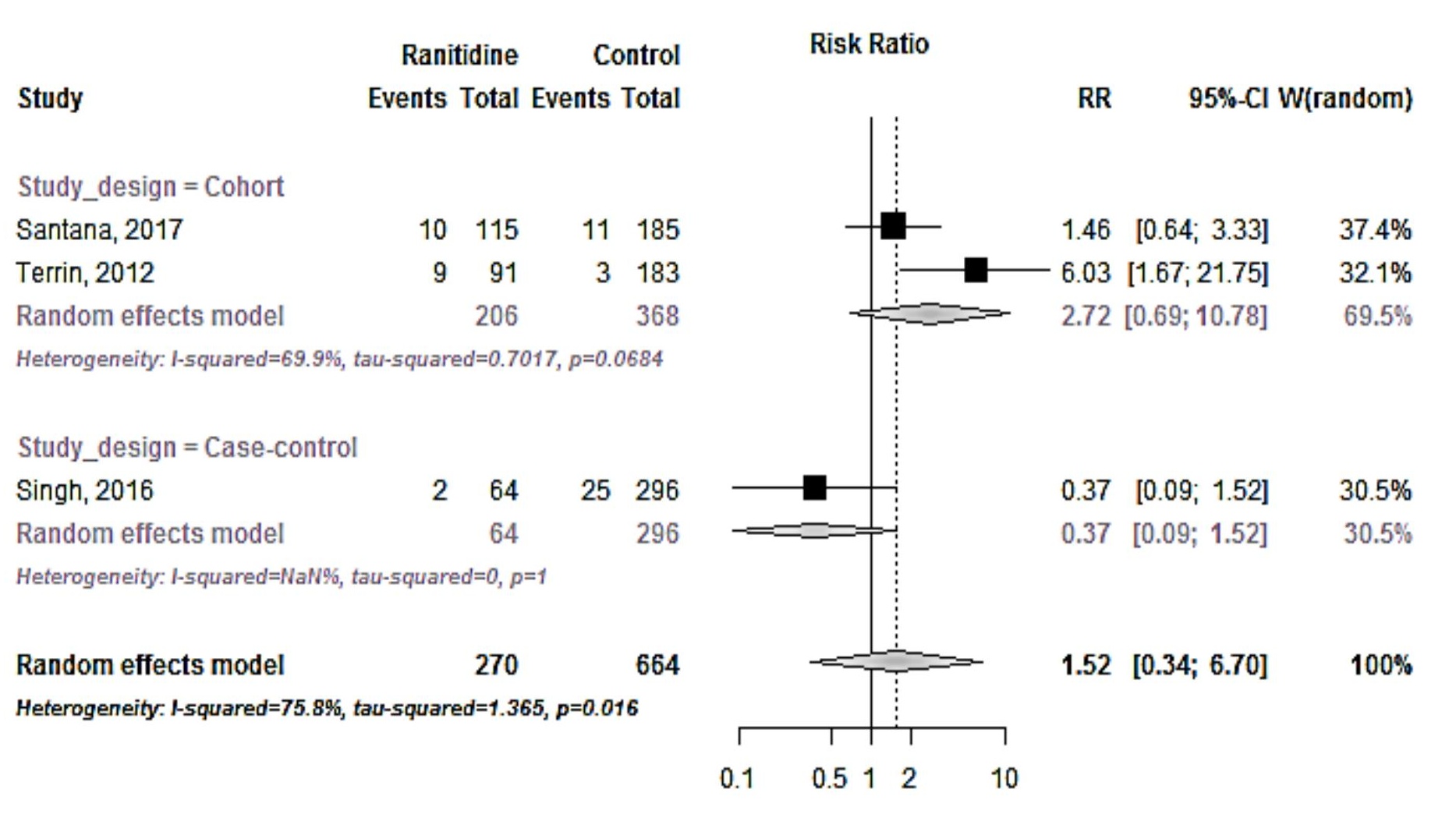

This is different to the others meta-analyses which reported association between NEC and the use of $\mathrm{H}_{2} R A$ by hospitalized neonates (pooled ORs: $2.81,95 \% \mathrm{Cl}$ : 1.19-6.64, $R$ : $0 \%)$. The $\mathrm{H}_{2} \mathrm{RA}$ use was not associated with the risk of death (pooled OR: $2.40,95 \% \mathrm{Cl}$ : $0.48-$ 11.96, R: $83 \%$ )

\section{Conclusions}

This meta-analysis has shown an association between the use of $\mathrm{H}_{2} \mathrm{RA}$ and the risk of infections in neonates, but not with NEC. Further safety studies including well defined patient groups are needed to increase the evidence for the safe use of H2RA in neonates and to support the development of guidelines by regulatory agencies. Cohort studies were more sensitive to identify differences. 